\title{
Impact of Feedback Channel Delay over Joint User Scheduling Scheme and Separated Random User Scheduling Scheme in LTE-A System with Carrier Aggregation
}

\author{
Ahmed A. Ali, Rosdiadee Nordin, Mahamod Ismail, and Huda Abdullah \\ Department of Electrical, Electronic and Systems Engineering, Universiti Kebangsaan Malaysia (UKM), Malaysia \\ Correspondence should be addressed to Ahmed A. Ali; bulebird_1010@yahoo.com
}

Received 17 March 2014; Accepted 15 May 2014; Published 12 June 2014

Academic Editor: Rui Zhang

Copyright (C) 2014 Ahmed A. Ali et al. This is an open access article distributed under the Creative Commons Attribution License, which permits unrestricted use, distribution, and reproduction in any medium, provided the original work is properly cited.

\begin{abstract}
In Long Term Evolution-Advanced (LTE-A), the signal quality in a wireless channel is estimated based on the channel quality measurements. The measurement results are used to select suitable modulation and coding scheme for each transmission, feedback, and processing delay, which can cause a mismatch between channel quality information (CQI) and current channel state at the base station. However, prospect delays in the reception of such CQI may lead to a system performance degradation. This study analyzes the impact of CQI feedback delay on joint user scheduling (JUS) scheme and separated random user scheduling (SRUS) scheme in LTE-A system over carrier aggregation. The analysis will be compared with the system having delayed channel and perfect knowledge at different deployment scenario. We will study the throughput performance of both scheduling schemes with different deployment scenario, and then recommend the suitable deployment scenario to keep the desired QoS for a specific number of users. Results show that, in main beam directed at sector boundaries and diverse coverage, JUS scheme performs better than SRUS, which can justify the intensive use of user equipment power and extra control signaling overhead.
\end{abstract}

\section{Introduction}

It has been suggested carrier aggregation (CA) as a technique in LTE-A, LTE-A system to enhance the data rate of the user [1-4]. In downlink LTE-A system could be developed up to $100 \mathrm{MHz}$ by aggregating several component carriers jointly the spectrum available for transmission [5]. The component carriers (CCs) can be contiguously or noncontiguously aggregated. Even though using CC can remarkably improve the users experienced throughput, it comes at the expense of extremely high channel state information (CSI) feedback overhead. The required feedback overhead increases proportion with the number of active CCs. This feedback overhead problem could severely limit the efficiency of the uplink communication and thus the CA system performance as a whole.

When the system is stretched from single carrier to multiple carriers, joint user scheduling schemes and separated random user scheduling schemes, termed as the JUS scheme and the SRUS scheme, respectively, are two straightforward schemes to manage the multiple carriers. If joint user scheduling algorithm is used, the eNB will calculate the throughput of users in each CC. It is the optimal scheduling algorithm. However, when the CCs are large and number of users, the complexity of the system is very high for the implementation. And if separated random user scheduling algorithm is adopted, the eNB calculates the user throughput in single CC, so the complexity is smaller than JUS algorithm.

Channel quality indicator plays a major role in the link adaptation process. It is a message sent by UE to e-Node-B characterizing the current downlink channel quality of the UE. It is measured from the reference symbols transmitted by e-Node-Bs. In the CQI measurement interval, resolution is measured in the frequency domain, reporting mechanisms, and so forth. They are all configurable parameters. These parameters have a massive impact on the system performance and their performance is studied, for example, in $[6,7]$. The delay occurs mainly due to the extended time that the 
physical layer takes for information processing at the end of eNode-B. In addition, the EU does not send CQI reports to all transmission time interval (TTI) [8]. Some of the CQI information generated is "aperiodic" in nature and can only be sent upon the user's request [9].

This paper analyses the performance of various CA scenarios, by using a system level simulator. The performance is evaluated in terms of per-UE average throughputs with CQI feedback delay on both scheduling schemes SRUS and JUS in CA deployment scenarios main beam directed at sector boundaries scenarios and diverse coverage scenarios. In particular, we investigate the impact of the CQI delay on the packet scheduling algorithm. It also examines the impact of the aperiodic reporting rate on the packet scheduling algorithm. Lastly, the paper recommends the suitable scenario, in which aperiodic CQI reports should be deployed to maintain the desired level of quality of service (QoS) for a given number of users. Accounting for up to 3-6 ms CQI delay, the maximum value of $6 \mathrm{~ms}$ is used, since that values more than $6 \mathrm{~ms}$ is not practical for LTE-A, captures the delays of the LTE-A feedback protocols and realistic base station processing. This study can provide an appropriate guideline for possible CA combinations and operations in upcoming beyond fourth generation mobile communications.

\section{Downlink and Uplink System Overview}

LTE-A is designed primarily for application in the time division duplex (TDD) and frequency division duplex (FDD). However, it can also be applied in a wide range of bandwidths. Aided by OFDMA, LTE-A has radio resource unit in frequency and time domains. The user is allocated a resource block (RB) in the downlink at each scheduling interval. The $\mathrm{RB}$ comprises 12 subcarriers in the frequency domain and one time slot of $0.5 \mathrm{~ms}$ duration in the time domain [8]. Since the scheduling interval takes $1 \mathrm{~ms}$, the RB is assigned to two consecutive time slots when it is assigned to the user. One RB assigned to the user contains orthogonal frequency division multiplexing (OFDM) symbols. Thus, one time long slot of $0.5 \mathrm{~ms}$ duration involves transmission of 7 OFDM symbols. Allowing some overhead for information control, an entity known as transport block (TB) carries single user data coming out at the physical layer (MAC layer) at the end of eNode-B [8].

Signal to interference noise ratio is measured by UE at each RB. This SINR is then converted into corresponding CQI which is based on [9] for which the picked-up TB and the block error rate (BLER) must be within $10 \%$. If there is a processing delay amounting to $3 \mathrm{~ms}$, then CQI reports sent to e-Node-B are used by the scheduler. Complying with LTEA standardization to prevent the scope of increased uplink signalling overhead due to UE-commenced channel state response this feedback reporting is completely monitored by eNode-B. A CQI feedback can be either aperiodic or periodic. In case of aperiodic CQI the feedback reports have more precise frequency domain channel state information than the periodic ones. Thus they allow the scheduler to make maximum use of frequency diversity. This however comes with an escalated cost due to more uplink signaling overhead.

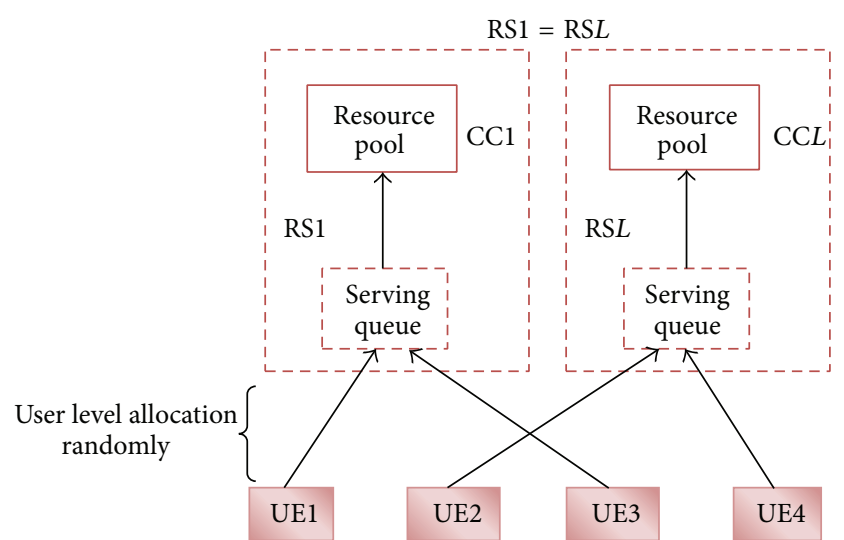

FIGURE 1: Illustration of the SRUS scheme.

\section{Proportional Fair Scheduling Algorithm}

The proportional fair scheduler is a mechanism that enables the functioning of multiuser systems through the principle of reliable time frequency. The time domain scheduling (TDS) systems are the basis for the profound existence of the proportional fair schedule. The schedule was applied to the LTE within TDS and frequency time scheduling systems to witness its OFDMA properties so as to enable harmonization data rate and overall system in multiuser mixture.

The carrier scheduling (CS) scheme which manages the resource of multiple CCs is one of the key factors that is indispensable to the LTE-Advanced system with CA [10]. There are two scheduling algorithms of proportional fair in LTE-A. Joint user scheduling and separated random user scheduling are two straightforward CS schemes [11].

3.1. Separated Random User Scheduling (SRUS). Compared with the JUS scheme, the SRUS scheme requires twolevel scheduling. The first level is responsible for allocating users to only one of the CCs. Here, a random sender is designed instinctively. Meanwhile, the load balance criterion is assumed to be guaranteed, which means that each CC must afford the transmissions of $U / C$ users in all (where $U$ is the number of users and $C$ is the number of component carriers). Four different users are allocated to two CCs randomly and uniformly; a simple allocation example is shown in Figure 1. In the second level the resource scheduling is controlled by the RSs. There should be the same number of RSs and CCs in the system. Consequently, the resource that is contained in each RS's resource pool is from only one of the CCs. Since only one CC is required for each user to communicate with, the SRUS scheme is very simple to apply. When using SRUS the UEs behaviour is in fact the same as that in the singlecarrier systems. Thus, for the UEs, it is not necessary to change anything. Therefore SRUS is the most basic CS scheme for the LTE-Advanced system with CA [11].

3.2. Joint User Scheduling (JUS). JUS is one of the explicit CS schemes to manage the multiple CCs. The RS is one of the multiuser schemes. It is an aggregate of RBs schemes, which 


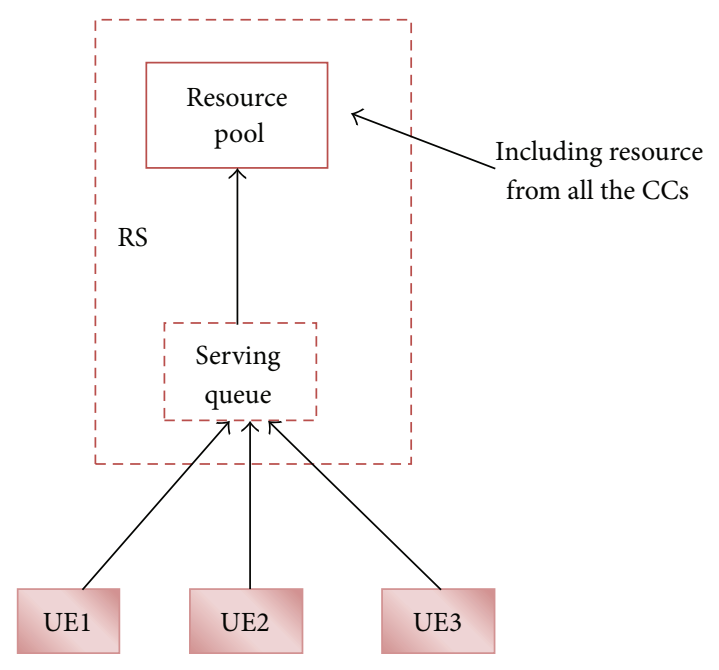

FIGURE 2: Illustration of the JUS scheme.

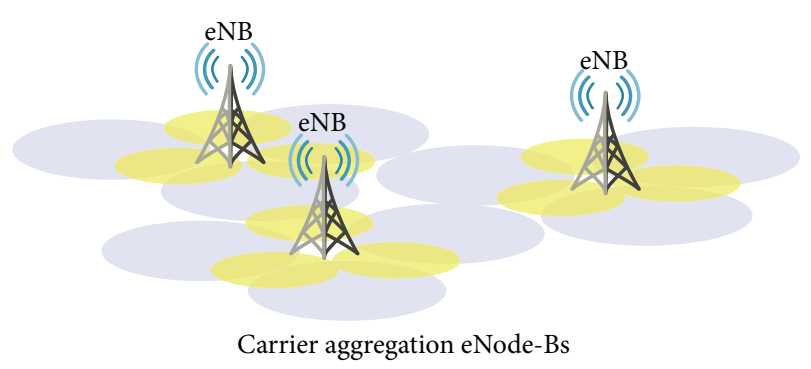

FIGURE 3: Main beam directed at a sector boundary deployment scenario.

are part of the CCs. This single RS scheme is functional when relying on the JUS schemes. As illustrated in Figure 2, JUS puts the RBs of all the CCs into the resource pool of one single RS. On the other hand, there is only one-level scheduler of JUS, which means the application of any specific allocation method used contrary to the SS scheme. In other words, the users in the system are likely to receive data transmitted from all the available CCs simultaneously, even though some users may use only one CC to transmit data [11].

\section{Deployment Scenarios in LTE-A}

With CA, different network deployment scenarios are possible [12]. In general, there are two CA scenarios in real LTEAdvanced deployment which have been studied in this work.

4.1. Main Beam Directed at Sector Boundaries. In some cases, the eNode- $B$ antennas used in various CCs may differ in beam direction patterns. The beam direction patterns are shifted across carriers at various sectors boundaries (Figure 3). Coverage for the CCs of the same eNode- $\mathrm{B}$ where CA is possible [13].

4.2. Diverse Coverage. In practice, spectrum allocation for an operator is frequently dispersed across various bands, having

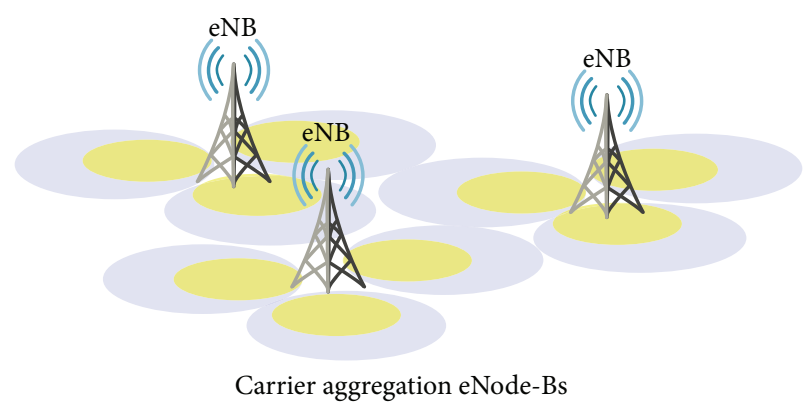

FIgURE 4: Diverse coverage deployment scenario.

large frequency separation. In such situations, coverage for a CC (of a higher frequency) may be smaller than that of other CCs (Figure 4). In both cases CA authorises higher user throughput at places where coverage of CCs overlaps [13].

\section{CQI Measurement}

The CQI helps in the selection of the correct modulation and coding scheme beneath various channel conditions. Also, it is applied in the calculation of priority metrics for the packet scheduling algorithms. The CQI consists of four basic steps in the simulator: (i) measuring SINR, (ii) introducing the measurement error to SINR, (iii) converting SINR values to the detached CQI steps, and (iv) giving CQI report. Ideal linear SINR is calculated for each physical resource block (PRB) $n$ from the received pilot power and total interference every measurement period. The measured linear SINR value for each PRB $n$. Each of the values derived from the measurement is converted to decibels as follows:

$$
\operatorname{SINR}_{\mathrm{dB}}(n)=10 * \log _{10}\left[\operatorname{SINR}_{\operatorname{lin}}(n)\right]+\text { Error }_{\mathrm{dB}} .
$$

A Gaussian distributed Error ${ }_{\mathrm{dB}}$ with parameter specified contrast and zero mean is introduced to the ideal SINR results. Quantization steps are applied on SINR values to convert them to detached CQI as follows:

$$
\mathrm{CQI}_{\mathrm{dB}}(n)=\mathrm{QStep}_{\mathrm{dB}} * \text { floor }\left(\frac{\operatorname{SINR}_{\mathrm{dB}}(n)}{\mathrm{QStep}_{\mathrm{dB}}}+0.5\right) \text {. }
$$

CQI is measured at specific time intervals with the longest multiples of TTI. The values derived from the measurement of CQI are reported using the CQI reporting scheme and are reported with certain delay. The basic CQI reporting scheme comprises both PRBs and comprehensive schemes and thus it requires application of advanced reporting techniques. However, the basic reporting scheme can be modified to suit the situation. For instance, it can be modified through changing the number of CQI reports per TTI. Full feedback reporting is achieved through measuring individual PRB values and reporting each of them separately. The average value of all PRBs (also known as wideband CQI) leads to the achievement of the least granularity.

Depending on specifications, each UE measures downlink channel quality. In addition, each of them selects the appropriate CQI values which show the modulation schemes, 
TABLE 1: Delays in LTE-A that input to the gross feedback delay.

\begin{tabular}{|c|c|c|}
\hline $\begin{array}{l}\text { Duration } \\
(\mathrm{ms})\end{array}$ & $\begin{array}{l}\text { Delay } \\
\text { type }\end{array}$ & Node and cause of delay \\
\hline 1 & Process & User: downlink channel estimation encode \\
\hline 1 & Transmit & $\begin{array}{l}\text { User: uplink data and channel state } \\
\text { information }\end{array}$ \\
\hline \multirow{2}{*}{1} & Transmit & BS: downlink data and pilots \\
\hline & Wait & Uplink subframe \\
\hline$\geq 1$ & Process & BS: resource allocation, encode \\
\hline \multirow{2}{*}{1} & Process & BS: uplink channel estimation, decode \\
\hline & Wait & Downlink subframe \\
\hline
\end{tabular}

the number of HS-PDSCH codes, and the transport block size. The CQI values must be selected in such a way that the transport block error prospect under the measured downlink channel condition does not exceed 0.1. After the suitable CQI values are selected, they are reported by UE to NodeB. Each of the values is reported at its own time. The UE's connection frame number (CFN) determines the CQI report timing in each case. CFN comes from the radio link creation procedure. A particular physical channel (HS-DPCCH) is used to transmit the CQI value. The CQI value has a field size of 20 bits on HS-DPCCH. The CQI value itself comprises 5 bits. Each CQI value is reported by a specific UE to Node-B [14].

\section{Feedback Delay}

The time lag taken between measuring the CSI and using it during the transmission process signifies the feedback delay. In most cases, the delay time comprises processing time, transmission time, and time taken when waiting for a scheduling grant. The delays happen at the user nodes or at the eNode- $\mathrm{B}$ and are contained in subframes. Table 1 gives an example (in summary) of the delays that occur on LTEA systems after the current 3GPP scheme [15]. Depending on how quickly the resource allocation issue is solved by the base station, the delay may change between 3 and 10 subframes. Most LTE-A systems have a delay of 6 to 10 subframes.

\section{Simulation Models}

We consider a downlink OFDM-based CA system, which is composed of a set of eNode-Bs and multiple UEs. The UEs are distributed in the cell randomly and united. In every $\mathrm{CC}$, there are $R$ resource blocks (RBs), each of which has $K$ subcarriers in frequency domain and one frame in time domain. In this study the traffic considered is continuous data stream (full buffer). Proportional fair (PF) scheduling algorithm is adopted; we consider this kind of scheduling because it pursues the maximum rate and meanwhile assure that none of terminals is starving. Adaptive modulation and coding (AMC) is employed in the transmission to achieve the high data rate. The UEs have the potential to estimate the channel quality information (CQI) of the downlink transmission and feed back it to the eNB.
TABLE 2: LTE-A simulation system configurations.

\begin{tabular}{ll}
\hline Parameter & Setting and value \\
\hline Carrier frequency & $2 \mathrm{Ghz}$ \\
Transmission bandwidth & $\begin{array}{l}20 \mathrm{Mhz} \text { per component carrier } \\
\text { Hexagonal grid, 7 cell sites, and 3 } \\
\text { sectors per eNode-B per } \\
\text { component carrier }\end{array}$ \\
Cell layout & $1 \mathrm{~ms}$ \\
Subframe (TTI) length & $2 \times 2$ MIMO with rank \\
MIMO configuration & Frequency domain based on PF \\
$\begin{array}{l}\text { Dynamic scheduling } \\
\text { algorithm }\end{array}$ & SRUS and JUS \\
CCs scheduling scheme & QPSK, 16-QAM, and 64-QAM \\
Modulation and coding & 1 TTI \\
scheme & Full buffer \\
Granularity of scheduling & 13 \\
Traffic model & 148 \\
$\begin{array}{l}\text { Number of UEs per sector } \\
\text { Resource elements for user } \\
\text { data apiece RB }\end{array}$ & $3-6$ ms \\
Feedback delay & 12 \\
Number of subcarriers apiece \\
RB
\end{tabular}

UE is similar to that in [9] in measures its SINR, except that the depends on the user's location and the intercell interference is dynamic in the cell. Using multipath fading, shadow, and pathloss, the immediate channel gain $\left(\operatorname{Gain}_{i \cdot j}(t)\right.$ of user $i$ at time $t$ on $\mathrm{RB} j$ ) is calculated. Then the immediate downlink SINR value of user $i$ at time $t\left(y_{i j}(t)\right)$ on $\mathrm{RB} j$ is computed using the approach used in [8] as shown below:

$$
y_{i \cdot j}(t)=\frac{p_{\text {total } * \operatorname{Gain}_{i j}(t)}}{N\left(I+N_{0}\right)} .
$$

The number of RBs available is the thermal noise and the total eNode-B downlink transmit power. UE location is dependent on intercell interference. Based on link to system level mapping, the SINR at each RB is converted to CQI value. UE channel conditions per RB formulate the UE CQI report in our simulation. As requested by eNode- $B$, this is reported periodically. Tuning can be the probability of this report happening at each TTI, and therefore the rate can be controlled. The CQI reporting rate (CRR) is identified as the probability of CQI report request generated per TTI. As per the assumptions of this paper, the scheduler uses the previously received report from the UE, as long as a new CQI report is not received from that UE (see Table 2).

\section{Simulation Results}

Simulation methodology based on $[12,16,17]$ has been adopted in order to identify the deployment scenario that fulfills the predefined QoS requirements on both scheduling schemes SRUS and JUS in LTE-Advanced system with carrier aggregation. Effective signal to interference plus noise ratio 


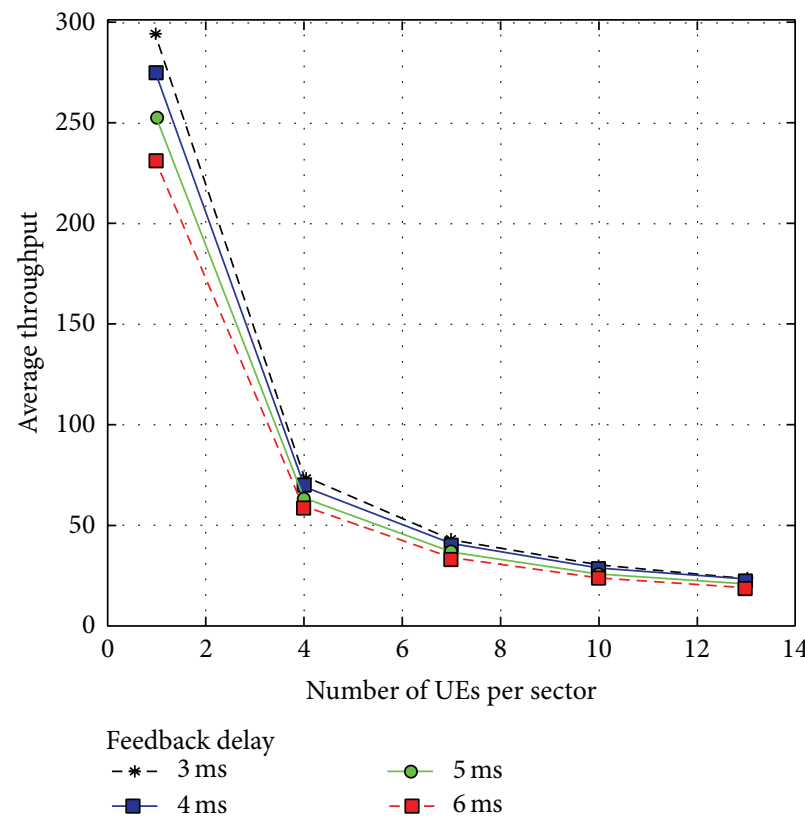

(a)

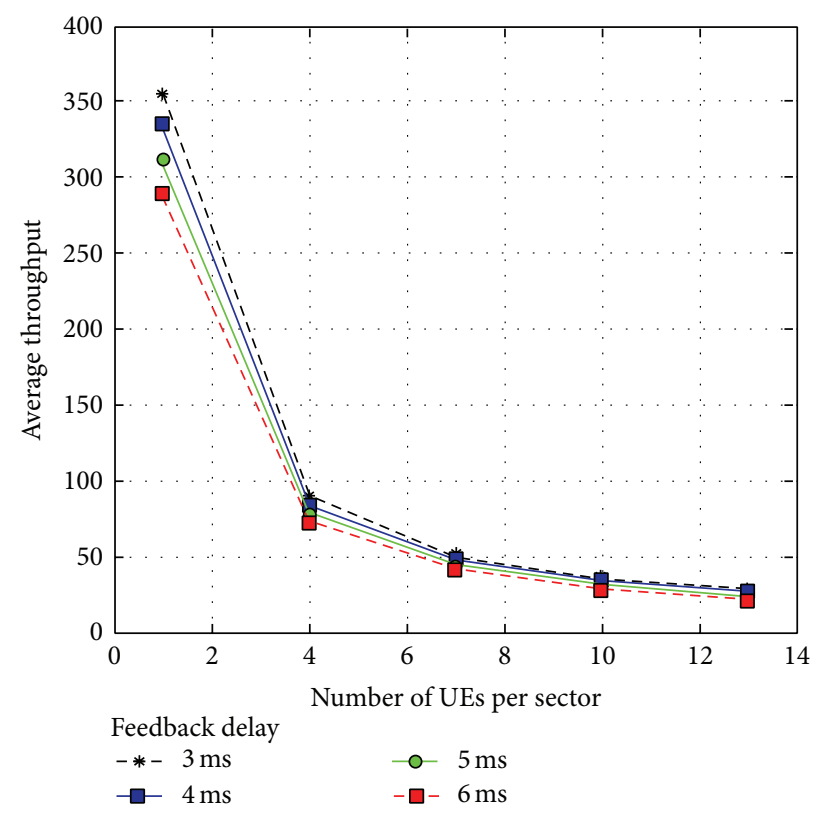

(b)

FIGURE 5: (a) Average throughput per user (SRUS). (b) Average throughput per user (JUS).

mapping (EESM) model is used to merge the SINR on each subcarrier to the RBs' SINR. Data for statistics are gathered from the whole network. With CA, different network deployments are possible [12]. In general, two CA scenarios in real LTE-Advanced deployment have been studied in this paper.

8.1. Main Beam Directed at Sector Boundaries Scenarios. In main beam directed at the sector boundary deployment scenario, the eNB antennas for different CCs can have various beam directions patterns. The directions of antenna beams are intentionally turned across carriers to improve throughput at sector boundaries. All CCs belong to the same 3GPP frequency band. In both noncontiguous and contiguous spectrum aggregation, CCs have the same propagation characteristics (fading, pathloss, and shadow).

Such resource allocation provides massive multiuser diversity gains. However requires the scheduler to strictly know the users' current CSI prior to the transmission. Extracting this information from the received signal is useless when fading and interference lead to different uplink and downlink channel states. With such asymmetric channels, we have to obtain the CSI from the users via feedback. Alongside (i) transmission errors, (ii) errors due to measuring and quantizing CSI at the user side, feedback adds, and (iii) feedback delay, high delay values may cause a wrong decision in the modulation level at the transmitter in uplink. Each of these three impairments causes a mismatch between the observed and the real CSI, which can substantially decrease the scheduler's performance. Since feedback delay primarily defines the upper bound of the achievable data rate in many mobile scenarios [18], we focus only on this impairment for now.
In order to identify the deployment scenario that fulfills the predefined QoS requirements in both scheduling schemes SRUS and JUS, we examine different feedback channel delay and the average user throughput with different number of LTE-A users per sector. Figures 5(a) and 5(b) show that the influence of the feedback channel delay on the average user throughput will be greater and it is clear how results get worse and degradation of performance is significant as the feedback channel delay increases. The decrease in average user throughput for the increase in feedback channel delay from practical point $3 \mathrm{~ms}$ to the $6 \mathrm{~ms}$ is about $21 \%$ in SRUS and $17 \%$ in JUS. Figure 5(b) shows that JUS has better performance in average user throughput under different feedback channel delay.

8.2. Diverse Coverage Scenarios. The diverse coverage deployment scenario, one of the most typical scenarios foreseen, is where eNB antennas are collocated and have the same beam patterns/directions for the CCs, providing approximately the same coverage on all CCs, two different 3GPP frequency bands used in the system. Used CCs have various propagation characteristics. In such instances, coverage for CCs of a higher frequency may be smaller than that of other CCs. This means more traffic load for CCs with lower frequency as they provide better channel quality. As seen in Figures 6(a) and 6(b) the decrease in average user throughput with increase in feedback channel delay is quite negligible. The decrease in average user throughput for the increase in feedback channel delay from practical point $3 \mathrm{~ms}$ to the $6 \mathrm{~ms}$ is about $18 \%$ in SRUS and 13\% in JUS. Figure 6(b) shows a remarkable increase in average user throughput with JUS scheme, under different feedback channel delay. JUS 


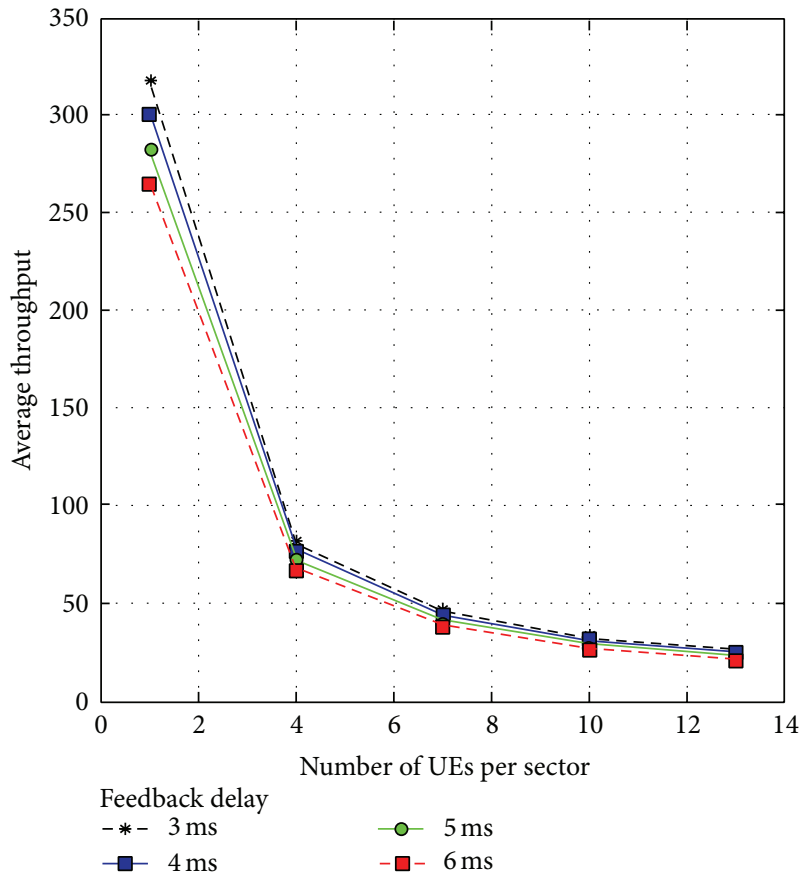

(a)

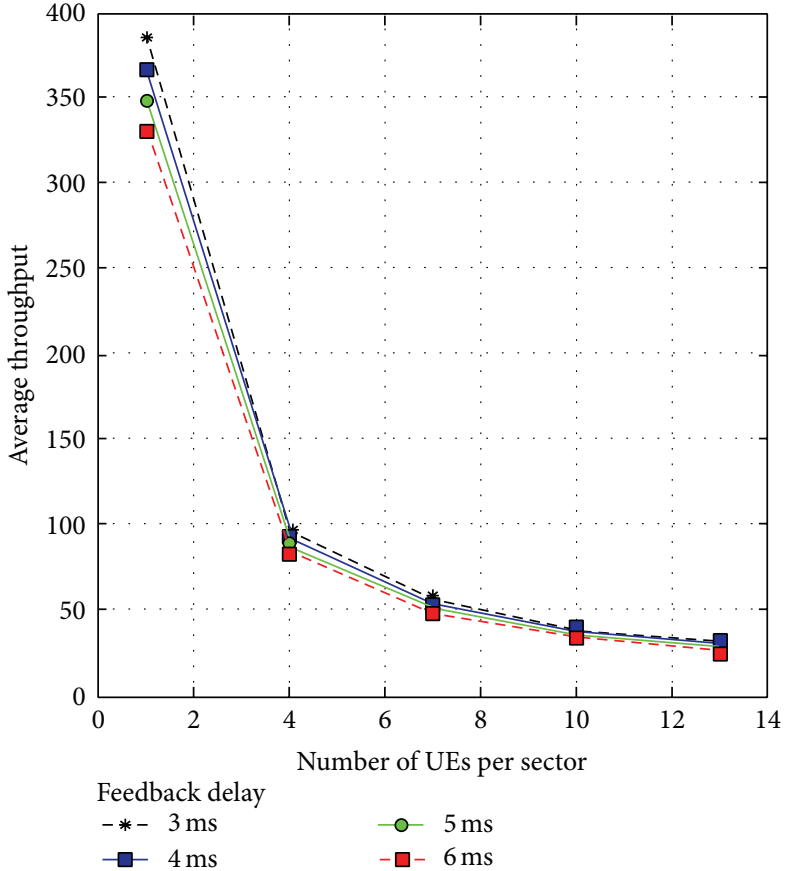

(b)

FIGURE 6: (a) Average throughput per user (SRUS). (b) Average throughput per user (JUS).

performs better than SRUS. These imply better performance of JUS scheme compared to SRUS scheme in diverse coverage deployment scenario.

Our results showed better performance of diverse coverage deployment scenario in comparson with main beam directed at the sector boundary deployment scenario. In addition, an improvement of average user throughput is clearly achieved with diverse coverage deployment scenario due to better channel quality in JUS and SRUS under this scenario. This would be a typical scenario when the CCs are of different bands or the same band. The degradation of diverse coverage deployment scenario performance due to feedback channel delay was far less than main beam directed at the sector boundary deployment scenario.

\section{Conclusion}

Along this paper the impact of CQI feedback delay on both scheduling schemes SRUS and JUS in LTE-Advanced system with carrier aggregation is addressed. An initial study was carried out as a specific analysis for CA scenarios in real LTE-Advanced deployment in order to assess the amount of performance degradation that can be attributable to feedback channel delay. It was found that the diverse coverage deployment scenario is more accurate with less variance than the main beam directed at the sector boundary deployment scenario. Similarly, diverse coverage deployment scenario showed less degradation in performance due to feedback channel delay compared to the main beam directed at the sector boundary deployment scenario. Diverse coverage appears to be optimal choice for both scheduling schemes.
It offers higher overall throughput and higher average user throughput than beam directed at sectors boundaries scenarios.

\section{Conflict of Interests}

The authors declare that there is no conflict of interests regarding the publication of this paper.

\section{Acknowledgments}

The authors would like to thank the anonymous reviewers who helped to improve the quality of this paper. The research work for this project is supported by Grant reference no. GUP-2012-036.

\section{References}

[1] G. Yuan, X. Zhang, W. Wang, and Y. Yang, "Carrier aggregation for LTE-advanced mobile communication systems," IEEE Communications Magazine, vol. 48, no. 2, pp. 88-93, 2010.

[2] M. Iwamura, K. Etemad, M.-H. Fong, R. Nory, and R. Love, "Carrier aggregation framework in 3GPP LTE-advanced," IEEE Communications Magazine, vol. 48, no. 8, pp. 60-67, 2010.

[3] Y. Wang, K. I. Pedersen, T. B. Sorensen, and P. E. Mogensen, "Carrier load balancing and packet scheduling for multi-carrier systems," IEEE Transactions on Wireless Communications, vol. 9, no. 5, pp. 1780-1789, 2010.

[4] K. I. Pedersen, F. Frederiksen, C. Rosa, H. Nguyen, L. G. U. Garcia, and Y. Wang, "Carrier aggregation for LTE-advanced: 
functionality and performance aspects," IEEE Communications Magazine, vol. 49, no. 6, pp. 89-95, 2011.

[5] 3GPP TR 36. 913 v8. 0. 0, " Requirements for further advancements for EUTRA (LTE-Advanced), 2008.

[6] K. I. Pedersen, G. Monghal, I. Z. KovÃącs et al., "Frequency domain scheduling for OFDMA with limited and noisy channel feedback," in Proceedings of the IEEE 66th Vehicular Technology Conference (VTC 2'07), pp. 1792-1796, Baltimore, Md, USA, October 2007.

[7] N. Kolehmainen, J. Puttonen, P. Kela, T. Ristaniemi, T. Henttonen, and M. Moisio, "Channel quality indication reporting schemes for UTRAN long term evolution downlink," in Proceedings of the IEEE 67th Vehicular Technology ConferenceSpring (VTC '08), pp. 2522-2526, Singapore, May 2008.

[8] A. Toskala and H. Holma, LTE For UMTS-OFDMA and SCFDMA Based Radio Access, John Wiley and Sons, 2009.

[9] 3GPP TS 36. 213 v8. 8. 0, "Evolved Universal Terrestrial Radio Access (E-UTRA):Physical layer procedures (Release 8)".

[10] L. Chen, W. Chen, X. Zhang et al., "Analysis and simulation for spectrum aggregation in LTE-Advanced system," in Proceedings of the IEEE 70th Vehicular Technologh Conference on Fall (VTC '09), pp. 1-6, Anchorage, AL, USA, September 2009.

[11] A. A. Ali, R. Nordin, and H. Abdullah, "Performance of Separate Random User Scheduling (SRUS) and Joint User Scheduling (JUS) in the Long Term EvolutionâĂŤAdvanced (LTE-A)," Journal of Theoretical and Applied Information Technology, vol. 59, no. 1, 2014.

[12] 3GPP, "Evaluation methodology for LTE-A Heterogeneous networks," R1-082554, 2008.

[13] 3GPP TS 36.300, “Overall description for Evolved- UTRA," V10. 4. 0, June 2011.

[14] H. A. M. Ramli, R. Basukala, K. Sandrasegaran, and R. Patachaianand, "Performance of well known packet scheduling algorithms in the downlink 3GPP LTE system," in Proceedings of the IEEE 9th Malaysia International Conference on Communications with a Special Workshop on Digital TV Contents (MICC '09), pp. 815-820, Kuala Lumpur, Malaysia, December 2009.

[15] 3GPP, "E-UTRA physical channels and modulation (release 9)," 3GPP, Technical Specification TS 36. 211 V9. 1. 0, 2010.

[16] 3GPP, "LTE-A Simulation Methodology and Assumptions," R1082610, 2008.

[17] J. C. Ikuno, M. Wrulich, and M. Rupp, "System level simulation of LTE networks," in Proceedings of the IEEE 71st Vehicular Technology Conference (VTC’10), Taipei City, Taiwan, May 2010.

[18] S. Valentin and T. Wild, "Studying the sum capacity of mobile multiuserdiversity systems with feedback errors and delays," in Proceedings of the IEEE 72nd Vehicular Technology Conference (VTC '10), Ottawa, Canada, September 2010. 

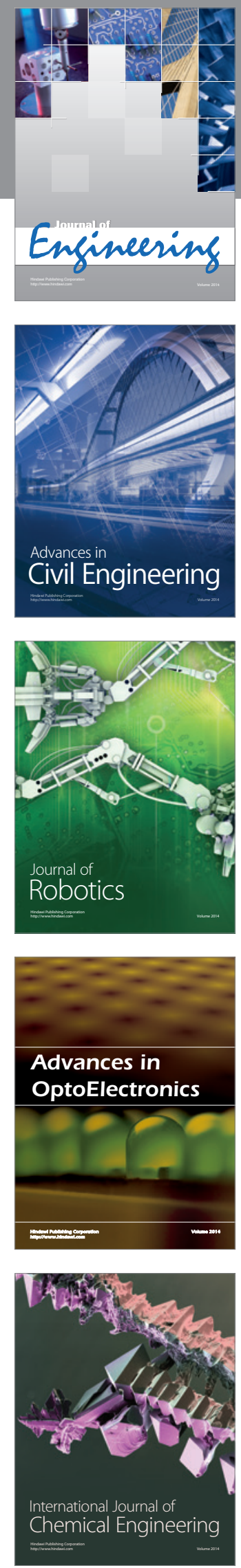

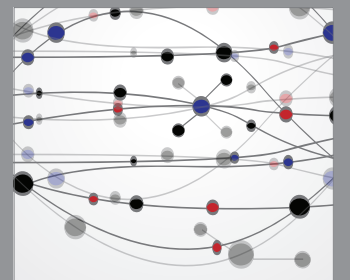

The Scientific World Journal
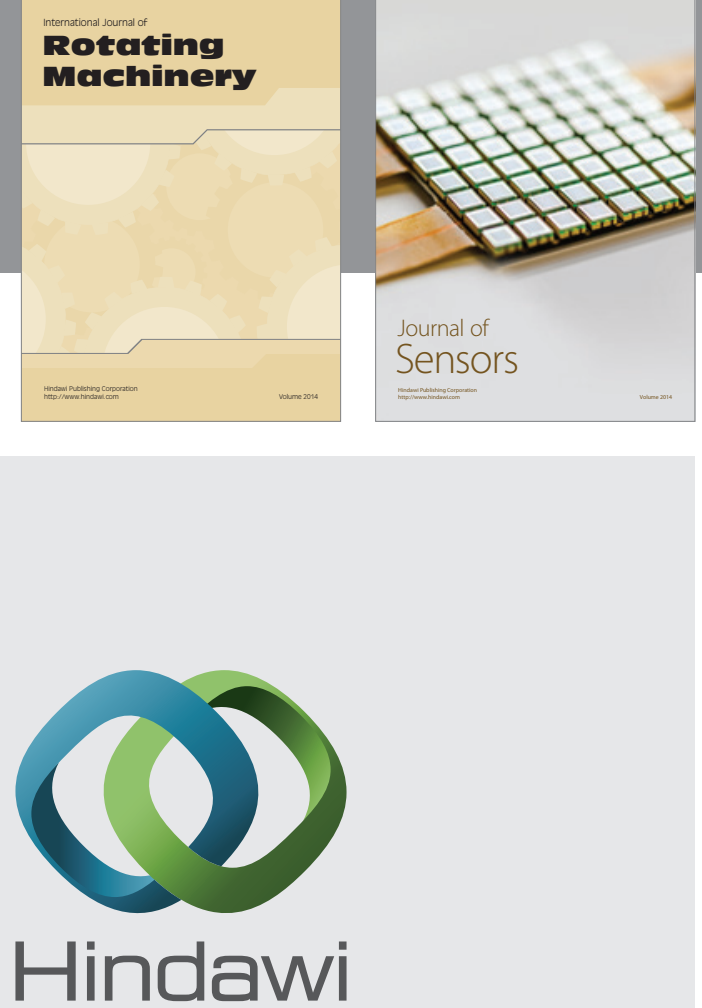

Submit your manuscripts at http://www.hindawi.com
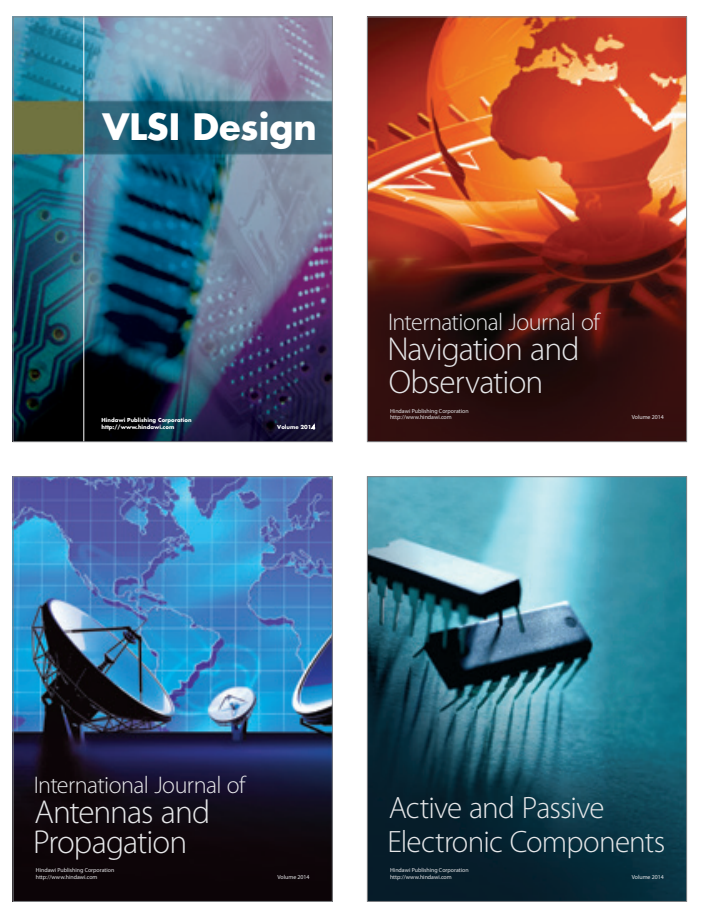
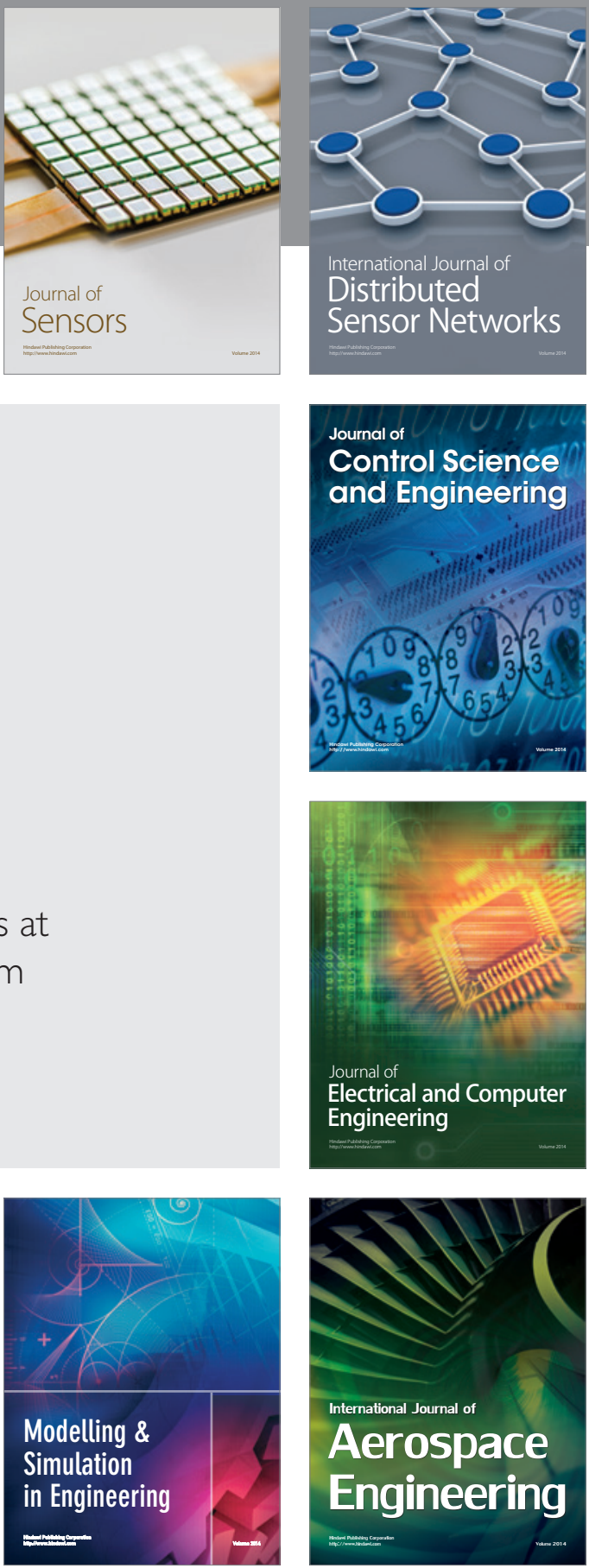

Journal of

Control Science

and Engineering
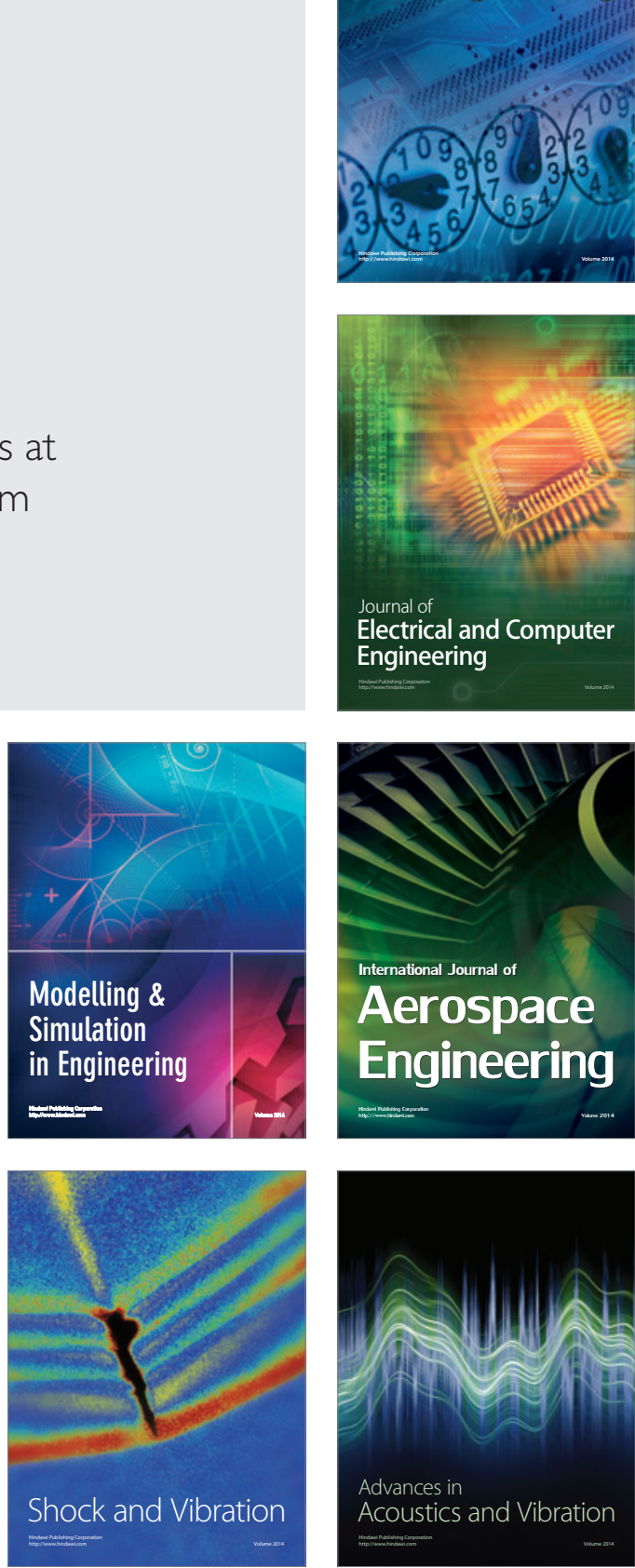